\title{
SINAL RADIOGRÁFICO DE ACÚMULO FECAL NO CECO PARA DIAGNÓSTICO DFERENCIAL DE APENDICITE AGUDA EM RE- LAÇÃO A OUTRAS DOENÇAS INFLAMATÓRIAS ABDOMINAIS
}

\author{
RADIOGRAPHIC SIGN OF FECAL LOADING IN THE CECUM FOR DIFFERENTIAL DIAGNOSIS OF \\ ACUTE APPENDICITIS IN COMPARISION WITH OTHER ABDOMINAL INFLAMMATORY DISEASES
}

Andy Petroianu1', Luiz Ronaldo Alberti², Renata Indelicato Zac ${ }^{3}$

\begin{abstract}
${ }^{1}$ Professor Titular do Departamento de Cirurgia da Faculdade de Medicina - UFMG, Pesquisador IA do CNPq. ${ }^{2}$ Cirurgião Geral e Pediátrico, Gastroenterologista. Mestre e Doutor em Cirurgia. ${ }^{3}$ Residente de Dermatologia da Santa Casa de Belo Horizonte. Instituto Alfa de Gastroenterologia do Hospital das Clínicas. Universidade Federal de Minas Gerais.

Av. Alfredo Balena, no 110. CEP 30130-100 / Belo Horizonte - MG.

Correspondência: Prof. Andy Petroianu. Av. Afonso Pena, no 1626, Apto 1901. CEP 30130-005, Belo Horizonte, Minas Gerais.

Telefone(Fax): (31) 3274-7744. e-mail: petroian@medicina.ufmg.br
\end{abstract}

Petroianu A, Alberti LR, Zac RI. Sinal radiográfico de acúmulo fecal no ceco para diagnóstico dferencial de apendicite aguda em relação a outras doenças inflamatórias abdominais. Medicina (Ribeirão Preto) 2007; 40 (4): 567-75, out./dez.

RESUMO: Objetivo: O objetivo do presente trabalho foi verificar um sinal radiográfico caracterizado pela imagem de acúmulo fecal no ceco, em radiografias simples de abdome em doentes com apendicite aguda, bem como avaliar a sensibilidade e especificidade desse sinal nessa afecção, em comparação com outras doenças inflamatórias agudas da parte direita do abdome. Método: Foram estudados prospectivamente 470 pacientes consecutivos, de ambos os sexos, internados com abdome agudo e dor localizada no flanco direito, distribuídos em quatro grupos: Grupo $1(n=170)$ - pacientes portadores de apendicite aguda, Grupo $2(n=100)$ - pacientes portadores de cálculo na via urinária direita, Grupo $3(n=100)$ - pacientes portadoras de afecções ginecológicas agudas à direita e Grupo $4(n=100)$ - pacientes operados por colecistite aguda. Radiografias simples de abdome foram realizadas em todos os casos dos quatro grupos durante a crise inflamatória aguda que precederam o tratamento. Resultados: A presença do sinal radiográfico de enchimento fecal esteve presente em 165 pacientes do Grupo 1, em 19 pacientes com cálculos nas vias urinárias, em 12 pacientes com afecções ginecológicas e em 13 pacientes com afecções das vias biliares. A sensibilidade do sinal radiográfico para apendicite aguda foi de $97,05 \%$ e sua especificidade foi de $85,33 \%$. O valor preditivo positivo do sinal radiográfico para apendicite aguda foi de $78,94 \%$ entretanto, seu valor preditivo negativo foi de $98,08 \%$. Conclusão: A imagem de acúmulo fecal no ceco em radiografia simples de abdome na incidência ântero-posterior associa-se a apendicite aguda. Esse sinal é incomum na presença de outras doenças inflamatórias abdominais agudas do lado direito do abdome. A ausência desse sinal radiográfico em paciente com suspeita de apendicite aguda deve alertar para a grande possibilidade de o diagnóstico ser diferente do pressuposto.

Descritores: Apendicite. Abdome Agudo. Radiografia. Apendicite Aguda. Ceco. Diagnóstico. 


\section{1- INTRODUÇÃO}

Dentre as manifestações do abdome agudo, a dor abdominal na fossa ilíaca direita (FID) é provavelmente um dos mais desafiadores problemas da Medicina, na medida em que confronta possibilidades amplas de doenças cirúrgicas e não-cirúrgicas. ${ }^{1,2,3} \mathrm{O}$ manejo adequado desses pacientes exige, portanto, uma definição diagnóstica precisa sobre a necessidade de instituir-se procedimento cirúrgico ou tratamento clínico. Essa decisão requer dados sobre a evolução do quadro clínico associados ao exame físico, avaliação laboratorial de rotina e exames de imagem. ${ }^{4}$ Dentre as afecções que podem provocar o abdome agudo da FID, a apendicite aguda destaca-se como a causa mais frequiente. ${ }^{3}$

A apendicite aguda é diagnosticada com base na avaliação clínica, contagem de leucócitos, estudos radiográficos abdominais e ultra-sonografia abdominal $^{1}$. Entretanto, a não perfeita acurácia desses métodos leva a erro diagnóstico inicial aceitável em até $20 \%$ dos pacientes com dor no flanco direito. ${ }^{5}$ Essa situação é mais freqüente em crianças, em mulheres e em idosos. ${ }^{5,6}$ Além dos casos de apendicite aguda que não são diagnosticados precocemente, cerca de $15 \%$ a 40 $\%$ de todas as apendicectomias resultam na remoção de um apêndice aparentemente normal. ${ }^{7,8,9}$

A incidência de apendicectomias sem evidência de apendicite chega a taxas maiores de $50 \%$ nas mulheres grávidas. ${ }^{10} \mathrm{O}$ retardo no diagnóstico pode resultar em ruptura apendicular e está associado a elevada morbidade e mortalidade, além de aumento nos custos terapêuticos. ${ }^{11}$ Da mesma maneira, os diagnósticos falso-positivos levam a apendicectomias talvez desnecessárias, que também contribuem para aumento da morbimortalidade. ${ }^{12}$

Apesar das publicações sobre esse tema, o inconsistente uso desse recurso diagnóstico na prática, mostra que o papel do exame radiográfico para o diagnóstico da apendicite aguda ainda não foi completamente investigado. ${ }^{13,14}$ Os achados radiográficos abdominais na apendicite aguda estão documentados na literatura e em alguns estudos retrospectivos. ${ }^{15,16}$ Brooks e Kiellen (1965) ${ }^{17}$ compilaram alguns desses achados como sendo de importância na avaliação clínica dos pacientes com dor abdominal: apendicolito ( $2 \%$ a $22 \%)$, gás no apêndice $(<2 \%)$, dilatação gasosa em alças de intestino delgado, com níveis hidroaéreos confinados ao quadrante inferior direito - íleo sentinela ( $15 \%$ a $55 \%)$, aumento da densidade de partes moles no quadrante inferior direito ( $12 \%$ a $33 \%$ ), deformidade do contorno cecal $(<5 \%)$, apagamento ou perda da faixa adiposa pré-peritoneal direita $(<8 \%)$, separação do conteúdo cecal da gordura pré-peritoneal direita $(<5 \%)$, abscesso, apagamento da margem do psoas (1\% a $8 \%)$, escoliose lombar côncava direita $(1 \%$ a $14 \%)$.

O objetivo do presente trabalho foi verificar a presença de imagem radiográfica de acúmulo fecal no ceco em radiografias simples de abdome de doentes com apendicite aguda. Esse sinal foi estudado comparativamente com outros pacientes com dor na fossa ilíaca direita em decorrência de outras doenças a fim de verificar sua acurácia.

\section{2- PACIENTES E MÉTOdo}

Esta pesquisa foi realizada de acordo com as recomendações da Declaração de Helsinque e da Resolução no 196/96 do Ministério da Saúde, sobre pesquisa envolvendo seres humanos ${ }^{18}$ e foi aprovada pelo Comitê de Ética em Pesquisa da Universidade Federal de Minas Gerais (UFMG). Todos os pacientes concordaram com a participação no estudo por meio de consentimento livre e esclarecido.

Foram estudados prospectivamente 470 pacientes consecutivos, de ambos os sexos, internados com abdome agudo e dor localizada no flanco direito, no Hospital das Clínicas da Universidade Federal de Minas Gerais (UFMG) e no Hospital Júlia Kubitschek da Fundação Hospitalar do Estado de Minas Gerais, entre janeiro de 2000 e dezembro de 2004 . Nenhum paciente que esteve de acordo com o presente protocolo foi excluído desta pesquisa.

Todos os pacientes foram identificados por idade, sexo e cor da pele (leucodérmicos, feodérmicos e melanodérmicos).

Cada doente recebeu o atendimento habitual para abdome agudo, que consistiu de anamnese; exame físico completo, incluindo o ginecológico nos casos em que houve dúvida sobre a origem da dor abdominal. Exames laboratoriais (hematológico, bioquímico de sangue, de urina), e de imagem (radiografia simples, ultra-sonografia e tomografia computadorizada de abdome) foram realizados dentro da rotina propedêutica. Radiografia simples de abdome em incidência ântero-posterior fez parte da avaliação complementar em todos os casos.

Os pacientes foram distribuídos em quatro grupos, de acordo com as seguintes afecções: 
Grupo 1 (n=170): Pacientes portadores de apendicite aguda, operados por essa doença, constituído por 101 pacientes do sexo masculino e 69 do sexo feminino com idade variando entre 5 dias e 73 anos (média de 31,33 \pm 14,27 anos), sendo 104 pacientes leucodérmicos, 54 feodérmicos e 12 melanodérmicos. Confirmou-se o diagnóstico pelo exame histopatológico dos apêndices. O critério histológico adotado para o diagnóstico da apendicite aguda foi a presença de infiltrado neutrofílico na camada muscular do apêndice, além de outros achados, dependendo da gravidade do caso. ${ }^{19}$ Esses pacientes foram submetidos a apenas um estudo radiográfico do abdome poucas horas antes do tratamento cirúrgico, como parte da rotina propedêutica para abdome agudo.

Os apêndices retirados dos pacientes do Grupo 1 foram classificados em quatro categorias ou fases da apendicite com base no aspecto morfológico ${ }^{19,20}$ : inicial ou catarral, fibrinopurulenta ou supurativa, gangrenosa, perfurativa.

Grupo 2 (n = 100): Pacientes de ambos os sexos, portadores de cálculo na via urinária direita (88 casos de nefrolitíase e 12 de ureterolitíase). O grupo foi constituído por 40 pacientes do sexo masculino e 60 do sexo feminino, com idade variando entre 4 e 84 (média de 40,82 $\pm 14,48$ ) anos, sendo 54 pacientes leucodérmicos, 37 feodérmicos e nove melanodérmicos. O diagnóstico foi confirmado em todos os casos por pelo menos um dos seguintes exames: radiografia simples de abdome, urografia excretora, ultra-sonografia e tomografia computadorizada. Todos realizaram a radiografia do abdome durante o episódio de dor intensa provocada pela litíase.

Grupo $3(\mathbf{n}=\mathbf{1 0 0})$ : Pacientes portadoras de afecções ginecológicas agudas à direita, constituído por mulheres com idade variando entre 16 e 76 (média de 32,60 $\pm 10,58$ ) anos, sendo 29 leucodérmicas, 49 feodérmicas e 22 melanodérmicas. O diagnóstico foi confirmado em todos os casos por exames que incluíram o clínico completo, ultra-sonografia pélvica, laparoscopia, além de verificação cirúrgica e estudo anatomopatológico, nos casos operados. As afecções que motivaram o atendimento foram hidrossalpingite aguda em 63 pacientes, gravidez tubária rota em 22, ruptura de cisto ovariano em 14 e torção de ovário em dois casos. Em uma paciente de 46 anos, foi verificada a presença de hidrossalpingite em associação a ruptura de cisto ovariano.Todas as doentes foram submetidas a estudo radiográfico simples do abdome como propedêutica do abdome agudo.

Grupo 4 (n = 100): Pacientes de ambos os sexos (30 pacientes do sexo masculino e 70 do sexo feminino) com idade variando entre 17 e 90 (média de $47,17 \pm 18,28)$ anos, sendo 35 leucodérmicos, 54 feodérmicos e 11 melanodérmicos, operados de colecistite aguda, com o diagnóstico confirmado em todos os casos por meio do ato operatório e do exame histológico da vesícula biliar removida. Em oito casos, foi evidenciada a presença de colangite concomitante à colecistite. Radiografias simples de abdome em incidência ântero-posterior foi realizada em todos os casos, durante a crise de colecistite aguda que precedeu o ato operatório.

O sinal radiográfico investigado foi a presença de imagem intraluminar do ceco, caracterizada por hipotransparência entremeada por múltiplos pequenos focos de imagens hipertransparentes. Esse aspecto semelhante a flocos é característico de acúmulo fecal no cólon. Tal imagem eventualmente continuava-se no cólon ascendente (Figura 1).

Todo o procedimento da técnica radiográfica utilizada neste trabalho seguiu as recomendações de rotina para o estudo radiográfico do abdome, não expondo o paciente a riscos fora do habitual em toda propedêutica para abdome agudo. ${ }^{21}$

Para a avaliação estatística foi utilizado o método descritivo de média e desvio padrão da média para a idade dos pacientes. Para comparar a presença do sinal radiográfico nos quatro grupos estudados, utilizou-se o teste qui ao quadrado. As diferenças foram consideradas significativas para valores correspondentes a $\mathrm{p}<0,05{ }^{22}$

Com o objetivo de validar a presença do sinal radiográfico e confirmar seu valor na prática clínica, convidamos um professor de Radiologia da Faculdade de Medicina da Universidade Federal de Minas Gerais, para verificar a presença desse sinal nas radiografias deste estudo. Outros médicos que foram responsáveis pelos pacientes também foram convidados a verificar a presença desse sinal radiográfico. Considerou-se apropriada a análise por múltiplos profissionais, para validar a presença desse sinal como método propedêutico válido no diagnóstico da apendicite aguda.

Para a avaliação da acurácia do sinal radiográfico nas diferentes doenças, foram calculados a sensibilidade, a especificidade, o valor preditivo positivo (VPP) e o valor preditivo negativo (VPN) em todos os grupos. A concordância inter-observador do sinal radiográfico em estudo foi realizada pelo método Kappa. 

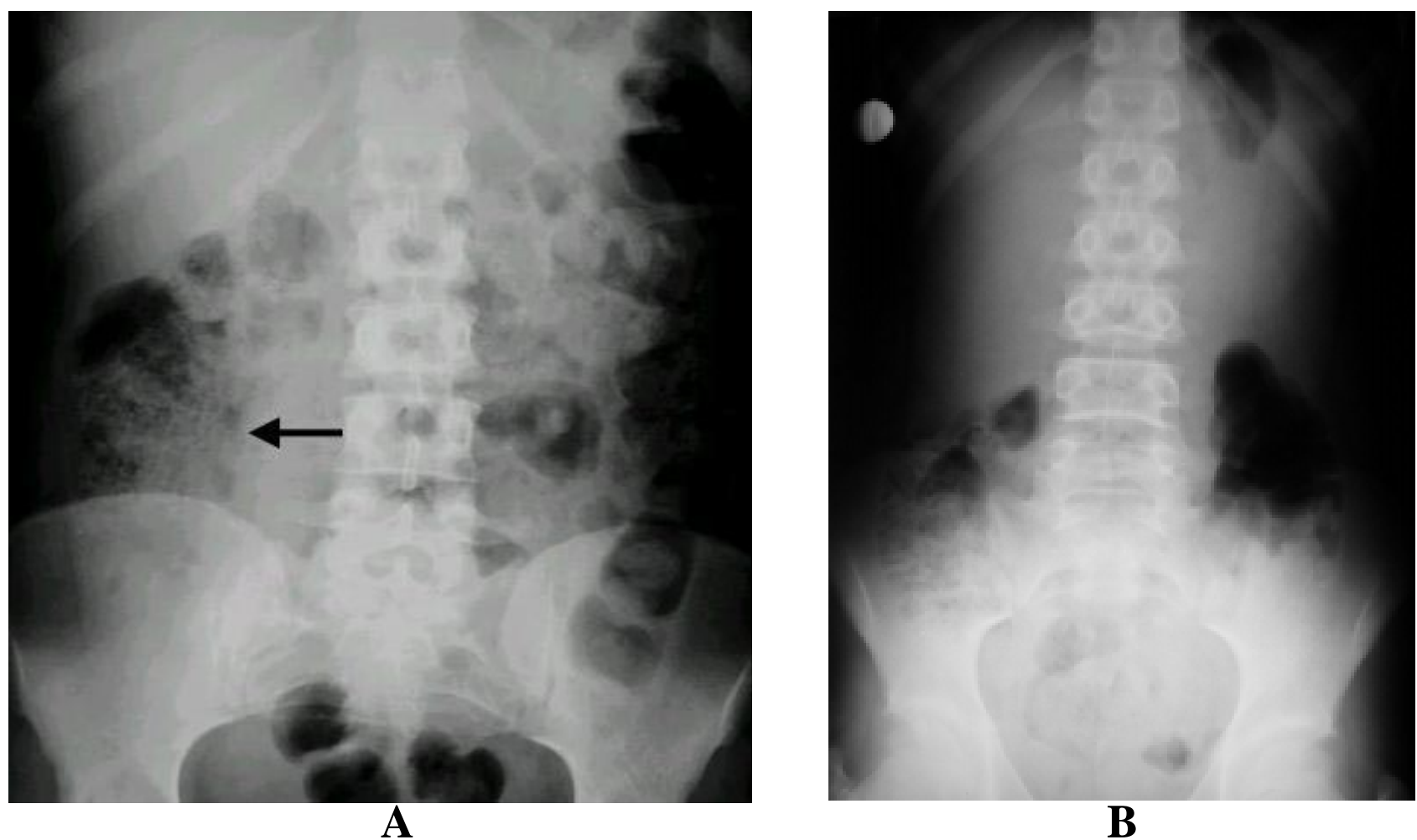

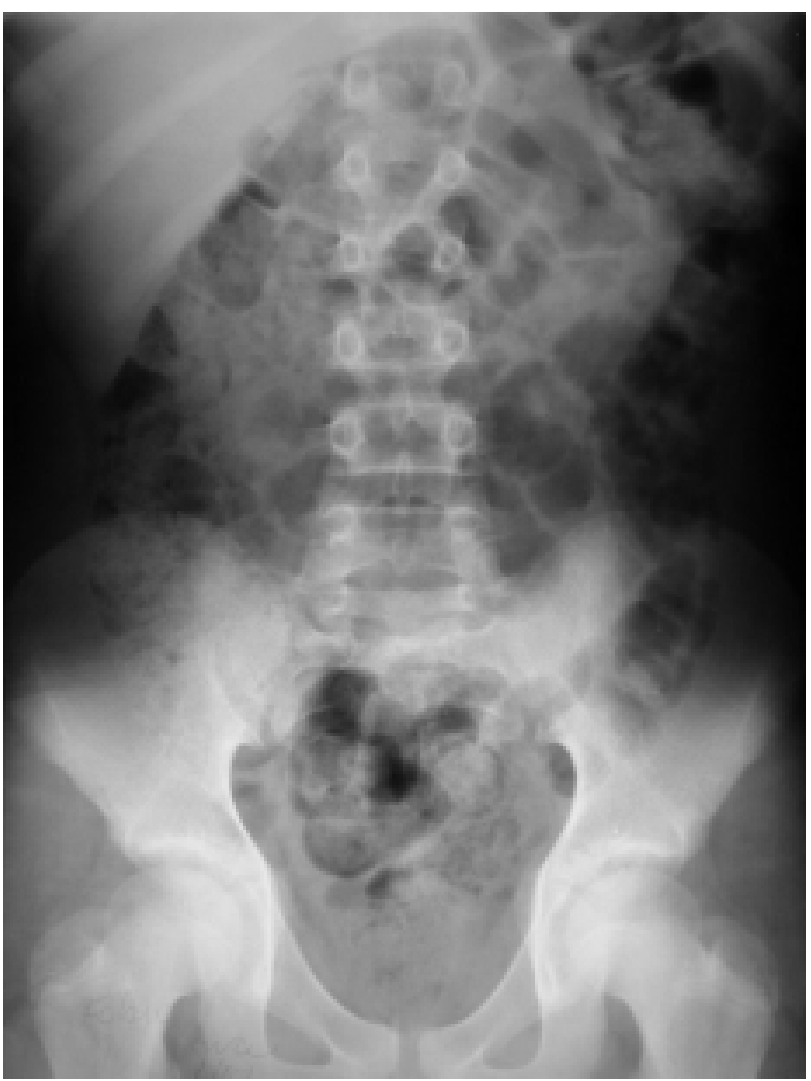

C

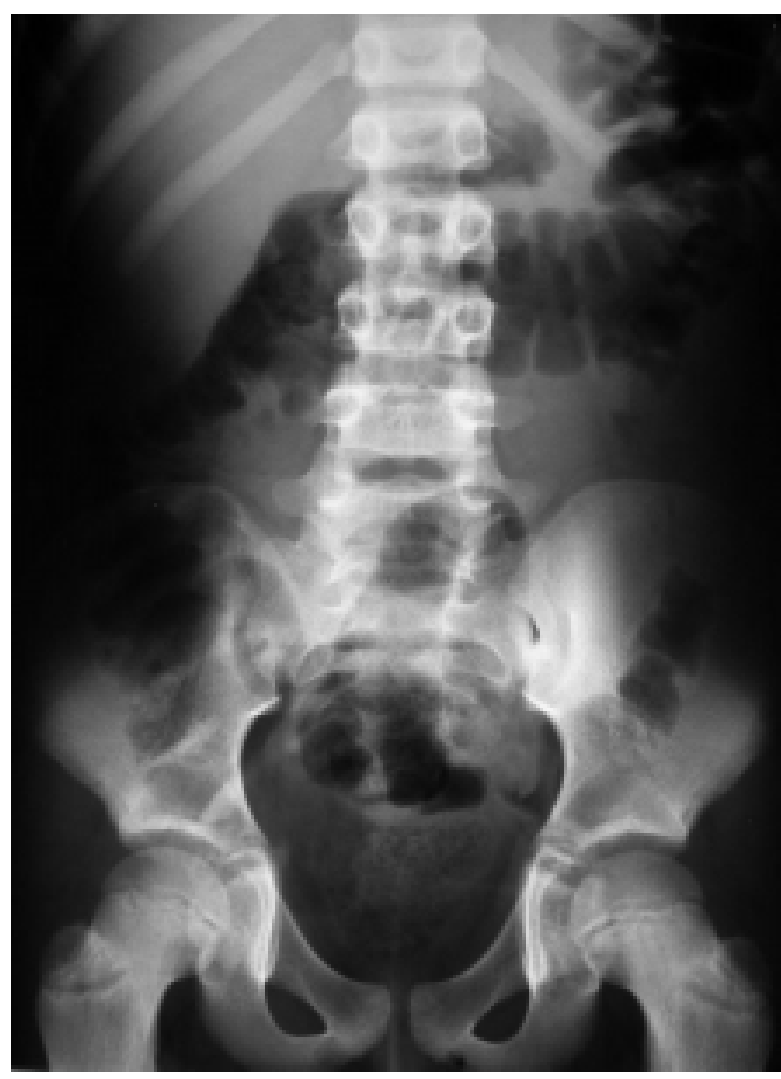

D

Figura 1: Radiografias simples de abdome, em incidência antero-posterior e ortostatismo. a, b, c: Pacientes com apendicite aguda. Observar imagem do ceco, que está distendido pelo acúmulo fecal (seta). d: Paciente normal, sem apendicite. 


\section{3- RESULTADOS}

A Tabela I mostra a presença do sinal radiográfico nos quatro grupos estudados. Observa-se que há diferença com significância estatística para a presença do sinal radiográfico de acúmulo fecal no ceco no Grupo 1 em relação aos demais grupos ( $<<0,0001)$. Não houve diferença nessa incidência entre os grupos 2,3 e 4 .

O sinal radiográfico de acúmulo fecal no ceco esteve presente em 165 pacientes portadores de apendicite aguda e ausente em apenas 5 casos (Tabela I). A sensibilidade do sinal radiográfico para apendicite aguda foi de $97,00 \%$ e sua especificidade foi de $85,33 \%$. O valor preditivo positivo desse sinal para apendicite aguda foi de 78,94\% e seu valor preditivo negativo foi de $98,08 \%$. (Tabela II)

Não houve diferença quanto a presença ou ausência do sinal radiográfico em relação ao sexo, cor da pele ou faixa etária $(\mathrm{p}>0,05)$. Também não houve tendência de ele ser mais encontrado em algum estádio histológico específico da apendicite. Assim, 23 estavam na fase catarral, 99 na fase fibrinopurulenta, 31 na gangrenosa e 17 na perfurativa.

O sinal radiográfico somente não esteve presente em quatro pacientes, com apendicite fibrinopurulenta e um em fase gangrenosa. Esse achado mostra que o sinal radiográfico incide em todas as fases da apendicite, tanto nas iniciais quanto mais avançadas.

Houve 49 pacientes na faixa etária pediátrica. Excetuando-se um paciente de oito anos, foi verificado a presença do sinal radiográfico em todas as outras crianças. Esse sinal foi verificado inclusive em um recém-nascido pré-termo com cinco dias de vida, cuja laparotomia para o diagnóstico do abdome agudo mostrou apendicite aguda perfurada.

O sinal radiográfico estudado foi encontrado em apenas 19 casos no Grupo 2. (Tabela I). Dos pacientes portadores de cálculo renal que apresentaram o sinal radiográfico (19 casos), dez eram do sexo masculino e nove do feminino, 17 eram portadores de nefrolitíase e dois eram portadores de ureterolitíase. Com relação à cor da pele, nove eram leucodérmicos, sete feodérmicos e três melanodérmicos. Não foi possível identificar característica de sexo $(\mathrm{p}=0,214)$, localização do cálculo urinário $(\mathrm{p}=0,827)$ ou cor da pele $(\mathrm{p}=0,482)$ que se relacionasse ao sinal radiográfico. A sensibilidade do sinal radiográfico para cálculos urinários foi de $19,00 \%$ e sua especificidade foi de
48,64\%. O valor preditivo positivo desse sinal para cálculos urinários foi de 9,09\%e seu valor preditivo negativo do sinal radiográfico para cálculos urinários foi de $68,96 \%$. (Tabela II)

O sinal radiográfico estudado foi encontrado em apenas 12 casos no Grupo 3. (Tabela I). Das pacientes portadoras de afecções ginecológicas que apresentaram o sinal radiográfico, quatro eram leucodérmicas, seis feodérmicas e duas melanodérmicas. Dessas pacientes, nove eram portadoras de hidrossalpingite, duas tiveram gravidez tubária rota e uma ruptura de cisto ovariano. Não foi possível identificar característica de cor da pele $(p=0,874)$ ou afecção ginecológica $(p=0,672)$ que se relacionasse com o sinal radiográfico. A sensibilidade do sinal radiográfico para afecções ginecológicas foi de $12,00 \%$ e sua especificidade foi de $46,75 \%$. O valor preditivo positivo desse sinal para afecções ginecológicas foi de $5,74 \%$ e seu valor preditivo negativo foi de $67,57 \%$.

O sinal radiográfico esteve presente em apenas 13 pacientes no Grupo 4. (Tabela I) Dos pacientes portadores de afecções das vias biliares que apresentaram o sinal radiográfico (13 casos), três eram do sexo masculino ( $10 \%$ dos homens) e dez do feminino (14,28\% das mulheres). Um desses pacientes teve colangite aguda associada à colecistite aguda. Com relação a cor da pele, cinco eram leucodérmicos e oito feodérmicos. Não foi possível identificar característica de sexo $(p=0,561)$ ou cor da pele $(p=0,396)$ relacionada ao sinal radiográfico. A sensibilidade do sinal radiográfico para afecções biliares foi de $13,00 \%$ e sua especificidade foi de $47,02 \%$. O valor preditivo positivo desse sinal para afecções biliares foi de $6,22 \%$ e seu valor preditivo negativo foi de $67,96 \%$. (Tabela II).

Os autores, o professor de Radiologia e os outros médicos convidados para avaliar as radiografias concordaram que o sinal radiográfico esteve presente em pacientes com apendicite. No entanto, esse sinal não foi constatado em cinco pacientes. Houve dúvida quanto à presença desse sinal em outros cinco pacientes, devido principalmente a superposição do osso ilíaco direito sobre a imagem do ceco $($ Kappa $=\mathbf{0 , 6 5 1})$ (CI $95 \%=0,501-0,801)$.

\section{4- DISCUSSÃO}

Apesar do avanço tecnológico e de conhecimentos, o diagnóstico da apendicite aguda ainda per- 
Tabela I: Número de pacientes com abdome agudo direito e imagem de acúmulo fecal no ceco em radiografia simples de abdome

\begin{tabular}{lccc}
\hline & & \multicolumn{2}{c}{ Sinal radiográfico } \\
Doença & $(n)$ & Presente & Ausente \\
\hline Apendicite aguda (Grupo 1)* & 170 & 165 & 5 \\
Cálculos urinários (Grupo 2) & 100 & 19 & 81 \\
Afecções ginecológicas (Grupo 3) & 100 & 12 & 88 \\
Afecções biliares (Grupo 4) & 100 & 13 & 87 \\
\hline
\end{tabular}

* Diferente em relação aos grupos 2,3 e 4 ( $p<0,0001)$

Tabela II: Comparação entre a acurácia dos valores estatísticos do sinal de acúmulo fecal no ceco nos quatro grupos de pacientes com abdome agudo do lado direito

\begin{tabular}{lcccc}
\hline Grupo & $\begin{array}{c}\text { Sensibilidade } \\
(\%)\end{array}$ & $\begin{array}{c}\text { Especificidade } \\
(\%)\end{array}$ & \multicolumn{2}{c}{ Palor preditivo } \\
1- Apendicite aguda & $97,00 *$ & $85,33 *$ & $78,94 *$ & Negativo (\%) \\
2- Cálculos urinários & 19,00 & 48,64 & 9,09 & $98,08 *$ \\
3- Afecções ginecológicas & 12,00 & 46,75 & 5,74 & 68,96 \\
4- Afecções biliares & 13,00 & 47,02 & 6,22 & 67,57 \\
\hline
\end{tabular}

* Diferente em relação aos grupos 2,3 e 4 ( $p<0,0001)$.

manece um desafio, principalmente em crianças, idosos e no sexo feminino, sabendo que retardo no tratamento, leva ao aumento da morbidade. ${ }^{11,23}$ Cabe ainda ressaltar que cerca de um terço das pessoas portadoras de apendicite aguda são submetidas à intervenção cirúrgica com diagnóstico pré-operatório incerto. A morbidade e mortalidade da apendicite aguda decorrem quase que exclusivamente da perfuração, relatada na literatura em proporções que variam entre $23 \%$ e $73 \% .^{23 / 26}$

Apesar de cálculos nas vias urinárias direitas não provocarem um quadro de inflamação peritoneal, assim como ocorre na apendicite aguda, na colecistite aguda e na doença inflamatória pélvica, considerouse pertinente acrescentar esse grupo por suas características de diagnóstico diferencial em dor apendicular. Dessa forma, avaliou-se também a influência da dor no acúmulo fecal dentro do ceco.

A incidência de perfuração apendicular nesta casuística foi inferior à descrita na literatura. Pena et al. $(2002)^{27}$ submeteram 1338 pacientes a um protocolo de avaliação da dor abdominal aguda que incluía ultra-sonografia seguida por tomografia computadorizada do abdome e obtiveram 15,5\% de apendicite agu- da na fase perfurada, enquanto na pesquisa atual a incidência foi de $8 \%$ para os pacientes do Grupo 1 . Esses dados indicam que talvez a precocidade do diagnóstico de apendicite na presente casuística tenha evitado que a apendicite chegasse à fase de perfuração. Nesse sentido, o sinal radiográfico contribuiu para essa condição favorável, pois em vários casos a sua presença foi decisiva para indicar-se a operação corretamente. ${ }^{23,24,27}$

Poucos estudos na literatura relataram não haver vantagens no estudo radiográfico do abdome na rotina propedêutica da apendicite aguda. ${ }^{28,29}$ Entretanto, nenhum deles se ateve ao estudo de pacientes apenas com dor abdominal na FID. Boleslawski et al. (1999) $)^{1}$, em pesquisa com 104 pacientes, consideraram a suspeita de apendicite aguda na presença de dois sinais radiográficos: apendicolito e alça ileal dilatada (alça sentinela) na FID, que ocorriam em $28 \%$ das apendicites agudas. Segundo esses autores, a radiografia simples de abdome modificou a suspeita diagnóstica e a conduta terapêutica em apenas $5 \%$ dos pacientes e não reduziu o número de retiradas de apêndices aparentemente sem alterações. Oncel et al. $(2003)^{30}$ descreveram que a radiografia simples de 
abdome ajudou no diagnóstico clínico em menos de $10 \%$ das apendicites, sendo que a maioria delas estava em fase avançada e com perfuração. A sensibilidade do sinal radiográfico de acúmulo fecal no ceco e no cólon ascendente, que foi estudado no presente trabalho $(97,05 \%)$, foi superior à descrita para outros sintomas e sinais de apendicite. . $^{1,30}$

Apesar do número maior de homens com apendicite, houve maior incidência de perfuração apendicular nas mulheres. Esse dado está em acordo com a literatura, que refere uma maior dificuldade para o diagnóstico da apendicite aguda em mulheres, em decorrência da dor abdominal relacionar-se a maior variedade de causas, incluindo ovulações dolorosas, doenças dos ovários, afecções das tubas uterinas e do útero, além de infecções do trato urinário, que são mais comuns em mulheres..$^{30,31}$ Em decorrência dessa dificuldade, houve provavelmente retardo no diagnóstico, propiciando a progressão do quadro apendicular até perfuração. Entretanto, com o sinal radiográfico estudado, que surge desde o início da apendicite, talvez essa doença possa ser tratada com menos demora.

Segundo Fenkins e Lee $(1970)^{32}$, não há sinal radiográfico patognomônico para o diagnóstico da apendicite aguda. Brady e Carrol (1957) (apud Fenkins e Lee, 1970) ${ }^{32}$ verificaram a associação entre apendicite aguda e o apendicolito em 24 de 74 casos de apendicite aguda estudados. Essa reduzida incidência tornou-se ainda menos destacada ao se encontrar esse sinal em outras doenças do sistema digestório e ginecológicas, além de ele poder ocorrer em pessoas normais.

Outro sinal relacionado à apendicite aguda é a presença de nível hidroaéreo no intestino delgado, próximo à região apendicular. Contudo, Oncel et al. $(2003)^{30} \mathrm{em} 162$ pacientes com apendicite aguda localizaram esse sinal em 34,7\% dos casos, incidência essa menor do que os $36,8 \%$ encontrados em pacientes com outras doenças. Por outro lado, o sinal radiográfico de inflamação cecal, além de ocorrer em uma incidência elevada nos pacientes com apendicite aguda, também é pouco comum em outras afecções inflamatórias, justificando sua importância para o diagnóstico da apendicite aguda.

Nesta casuística não houve erro de diagnóstico de apendicite aguda. Todos os apêndices estavam inflamados em diferentes estádios. Desde o início da pesquisa, houve alguns pacientes com dor no quadrante inferior direito do abdome, mas sem o sinal radiográfico estudado. Nesses casos, a operação foi adiada e propedêutica suplementar bem como o acompanha- mento por um tempo mais prolongado definiram os quadros abdominais como não apendiculares: ovulação dolorosa, linfadenite mesentérica, litíase urinária e dor sem causa identificada. Nenhum desses pacientes foi operado e a causa de dor foi resolvida com controle clínico.

Segundo Graham e Johnson $(1966)^{33}$, em pacientes com apendicite aguda em fases avançadas (gangrenosa ou perfurativa), pode-se detectar a presença de alteração radiográfica em apenas $62 \%$ dos casos. Por outro lado, a imagem de acúmulo fecal no ceco esteve presente em todos os casos na fase catarral e perfurativa e só esteve ausente em quatro dos 99 pacientes na fase fibrinopurulenta e um paciente na fase gangrenosa, mostrando sua constância em todas as fases morfológicas da apendicite, tanto iniciais quanto tardias.

Observou-se no presente trabalho, que o sinal de acúmulo fecal no ceco ocorreu em todos os pacientes acima de 40 anos, fato esse de importância maior, tendo em vista a elevada morbimortalidade da apendicite em decorrência de possível atraso terapêutico (Barnes et al. (1962). ${ }^{34}$ Cabe ainda ressaltar a possibilidade de a apendicite no idoso ter etiopatogenia diferente daquela do jovem. Mesmo se isso for verdade, o sinal radiográfico estudado é visto nos dois grupos sem diferença entre si.

Vários estudos radiográficos têm sido propostos para aumentar a acurácia diagnóstica na apendicite aguda, tais como: ultra-sonografia, tomografia computadorizada com contraste, tomografia helicoidal, cintilografia, ressonância nuclear magnética e ultra-sonografia com Doppler. Apesar do uso desses métodos imaginológicos, a acurácia diagnóstica para apendicite aguda ainda permanece abaixo de $95 \% .^{35,36}$

Ainda que o sinal descrito não seja patognomônico de apendicite aguda, ele fornece indícios muito confiáveis para o diagnóstico dessa afecção, especialmente se correlacionado aos achados do exame físico e laboratorial. O aspecto cirúrgico do ceco e eventualmente cólon ascendente confirmam que a imagem corresponde de fato a fezes. Essa imagem também poderia simular imagem de edema cecal, entretanto, a observação cuidadosa das radiografias, alguns exames tomográficos e a palpação do ceco durante algumas operações indicaram que o ceco não se apresentava inflamado e que havia conteúdo fecal em seu interior.

Novas pesquisas estão em andamento para confirmar a presença desse sinal radiográfico em apendicite aguda e explicar sua fisiopatologia, bem como definir a relação entre apendicite aguda e o acúmulo 
fecal no ceco. Uma hipótese que poderia eventualmente explicar esse fato seria o processo inflamatório da apendicite provocar íleo localizado no ceco. Como a função principal desse segmento colônico é absorver água, haveria conseqüente acúmulo de material fecal desidratado, que seria responsável pela imagem radiográfica descrita nesta pesquisa.

\section{5- CONCLUSÕES}

A imagem de acúmulo fecal no ceco em radiografia simples de abdome na incidência ântero-posterior associa-se a apendicite aguda.
Esse sinal é incomum em associação com outras doenças inflamatórias agudas do lado direito do abdome.

A ausência desse sinal radiográfico em paciente com suspeita de apendicite aguda deve alertar para a grande possibilidade de o diagnóstico ser diferente do pressuposto.

\section{6- AGRADECIMENTOS}

Os autores são gratos ao Professor Cid Sérgio Ferreira por seu auxílio na análise de radiografias deste trabalho.

Petroianu A, Alberti LR, Zac RI. Radiographic sign of fecal loading in the cecum for differential diagnosis of acute appendicitis in comparision with other abdominal inflammatory diseases. Medicina (Ribeirão Preto) 2007; 40 (4): 567-75, oct./dec.

ABSTRACT: Objective: The value of plain abdominal radiography in acute appendicitis has not been completely studied. Therefore, the purpose of this investigation was to verify a new radiographic sign: the presence of fecal loading in the cecum. Methods: 470 consecutive patients of both sexes admitted to the hospital due to acute abdominal pain localized in the right flank were prospectively studied. The patients were divided into four groups: Group $1(n=170)$-acute appendicitis - patients submitted to an abdominal radiographic study a few hours before surgical treatment; Group $2(n=100)$ - right nephrolithiasis submitted to an abdominal radiography during the episode of pain caused by the lithiasis; Group $3(n=100)$ - right acute inflammatory pelvic disease that underwent an abdominal radiographic study during the pain incident; Group $4(n=100)$ - acute cholecystitis submitted to abdominal radiography during the pain episode that preceded the surgical procedure. All of the radiographs were plain with an anteroposterior view of the abdomen. Results: The sign of fecal loading in the cecum was present in 165 patients of Group 1, in 19 patients of Group 2, in 12 patients of Group 3 and in 13 patients of Group 4. The sensitivity of the radiographic sign for acute appendicitis was $97,05 \%$ and its specificity was $85,33 \%$. The positive predictive value for acute appendicitis was $78,94 \%$ and its negative predictive value was $98,08 \%$. Conclusions: The radiographic image of fecal loading in the cecum is associated with acute appendicitis. This sign is uncommon in other acute inflammatory diseases of the right side of the abdomen. The absence of this sign makes the diagnosis of acute appendicitis improbable.

Keywords: Appendicitis. Abdomen, Acute. Radiography. Appendicitis, Acute. Cecum. Diagnosis.

\section{7- REFERÊNCIAS}

1 - Boleslawski E, Panis Y, Benoist S, Denet C, Mariani P, Valleur $P$. Plain abdominal radiography as a routine procedure for acute abdominal pain of the right lower quadrant. World $\mathrm{J}$ Surg 1999; 23:262-4.

2 - Marincek B. Nontraumatic abdominal emergencies: acute abdominal pain: diagnostic strategies. Eur Radiol 2002; 12: 2136-50.

3 - Zhou H, Yl-chen C, Jin-Zhe Z. Abdominal pain among children re-evaluation of a diagnostic algorithm. World J Gastroenterol 2002; 8: 947-51.
4 - Groselj-Grenc M, Repse S, Vidmar D, Derganc M. Clinical and laboratory methods in diagnosis of acute appendicitis in children. Croat Med J 2007; 48: 353-61.

5 - Reynolds SL. Missed appendicitis in a pediatric emergency department. Pediatr Emerg Care 1993; 9:1-3.

6 - McCALLION J, Canning GP, Knight RV. Acute appendicitis in the elderly, a five-year retrospective study. Age Ageing 1987; 16: $256-60$

7 - Kirshtein B, Bayme M, Domchik S, Mizrahi S, Lantsberg L. Complicated appendicitis: laparoscopic or conventional surgery? World J Surg 2007, 31: 744-9. 
8 - Deakin DE, Ahmed I. Interval appendicectomy after resolution of adult inflammatory appendix mass - is it necessary? Surgeon 2007, 5: 45-50.

9 - Petroianu A, Oliveira Neto JE, Alberti LR. Incidência comparativa da apendicite aguda em população miscigenada, de acordo com a cor da pele. Arq Gastroenterol 2004; 41: 24-6.

10 - Flum DR, Morris A, Koepsell T, Dellinger. Has misdiagnosis of appendicitis decreased over time? A population-based analysis. JAMA 2001; 286: 1748-53.

11 - Graff L, Russell J, Seashore J. False-negative and falsepositive errors in abdominal pain evaluation: failure to diagnose acute appendicitis and unnecessary surgery. Acad Emerg Med 2000; 7: 1244-55.

12 - Izbicki JR, Knoefel WT, Wilker DK. Accurate diagnosis of acute appendicitis: a retrospective and prospective analysis of 686 patients. Eur J Surg 1992; 158: 227-31.

13 - Davies DA, Yanchar NL. Appendicitis in the obese child. J Pediatr Surg 2007, 42: 857-61.

14 - Shimkin PM.Radiology of acute appendicitis. Am J Roentgenol 1978; 130: 1001-4.

15 - Beneventano TC, Clarence JS, Jacobson HG. The roentgen aspects of some appendiceal abnormalities. Am J Roentgenol Radium Ther Nucl Med 1966; 96: 344-60.

16 - Fagenberg D. Fecaliths of the appendix: incidence of significance. Am J Roentegenol 1963; 89: 752-9.

17 - Brooks DW, Killen DA. Roentgenographic findings in acute appendicitis. Surgery 1965; 57: 377-84.

18 - Petroianu A. Pesquisa em Medicina. In: Petroianu A, editor. Ética, moral e deontologia médicas. Rio de Janeiro: Guanabara Koogan; 2000. p.174-8.

19 - Crawford JM. Appendix. In: Cotran RS, Kumar V, Collins T, editors. Robbins- Pathologic basis of disease. 6th ed. Philadelphia: WB Saunders; 1999. p. 838-40.

20 - Jones MW, Paterson AG. The correlation between gross appearance of the appendix at appendicectomy and histological examination. Ann R Coll Surg Engl 1988; 70: 93-4.

21 - Bondrager KL. Abdome e procedimentos contrastados comuns. In: Bondrager KL, editor. Técnica radiológica e base anatômica. 3 ed. Rio de Janeiro: Guanabara Koogan; 2003. p. 211-50.

22 - Altman DG, Gardner MJ. Means and their differences. In: Altman DG, Machin D, Bryant TN, Gardner MJ, editors. Statistics with confidence. London: British Medical Journal Books; 2000. p. 28-35.
23 - Gracey D, McClure MJ. The impact of ultrasound in suspected acute appendicitis. Clin Radiol 2007, 62: 573-8.

24 - Adolph VR, Falterman KW. Appendicitis in children in the managed care era. J Pediatr Surg 1996; 31: 1035-7.

25 - Chou JS, Chung CR, Hu WM. Pain in the right lower quadrant. Am Fam Physician 2007, 15: 1541-2.

26 - Rappaport WD, Peterson M, Stanton C. Factors responsible for the high perfuration rate seen in early childhood appendicitis. Am Surg 1989; 55: 602-5.

27 - Pena BMG, Taylor GA, Fishman SJ, Mandl KD. Effect of an imaging protocol on clinical outcomes among pediatric patients with apendicitis. Pediatrics 2002; 110: 1088-93.

28 - Campbell JPM, Gunn AA. Plain abdominal radiographs and acute abdominal pain. Br J Surg 1988, 75: 554-7.

29 - Johansson EP, Rydh A, Riklund KA. Ultrasound, computed tomography and laboratory findings in the diagnosis of appendicitis. Acta Radiol 2007, 62: 573-8.

30 - Oncel M, Degirmenci B, Demirhan N, Hakemez B, Altuntas $Y E$, Aydinli $M$. Is the use of plain abdominal radiographs (PAR) a necessity for all patients with suspected acute appendicitis in emergency services? Curr Surg 2003; 60: 296-300.

31 - Dueholm S, Bagi P, Bud M. Laboratory aid in the diagnosis of acute appendicitis. Dis Colon Rectum 1989; 32: 855-9.

32 - Jenkins D, Lee P. Radiology in acute appendicitis. J Roy Coll Surg Edinb 1970; 15: 34-7.

33 - Graham AD, Johnson HF. The incidence of radiographic findings in acute appendicitis compared to 200 normal abdomens. Mil Med 1966; 131: 272-6.

34 - Barnes BA, Behringer GE, Wheelock FC, Wilkins EW. Treatment of appendicitis at the Massachusetts General Hospital (1937-1959). JAMA 1962; 180: 122-6.

35 - Birnbaum BA, Wilson SR - Appendicitis at the millennium. Radiology 2000, 215: 337-48.

36 - Incesu L, Yazicioglu AK, Selcuk MB, Ozen N. Contrast-enhanced power Doppler US in the diagnosis of acute appendicitis. Eur J Radiol 2004; 50: 201-9.

Recebido em 04/06/2007

Aprovado em 06/08/2007 